\title{
LARGE SCALE GRADIENT IN THE VELOCITY FIELD OF THE COMA CLUSTER AND A STUDY OF THE SPIN ORIENTATION OF GALAXIES IN THE VIRGO CLUSTER
}

\author{
M. IYE \\ National Astronomical Observatory, Tokyo 181, Japan \\ AND \\ T. OZAWA \\ Graduate University for Advanced Studies, Tokyo 181, Japan
}

This paper reports two studies on the distribution of the spin angular momenta of galaxies in physical clusters of galaxies.

i) Gradient in the Velocity Field of Coma Cluster

We studied the distribution of the line-of-sight velocity of galaxies in the Coma cluster by evaluating the dipole moment vector of the distribution. We compared the observed dipole moment vector with those obtained for 1000 Monte Carlo simulation runs where the observed line-of-sight velocities were reshuffled among the observed galaxies. We conclude that the Coma main cluster has a significant large scale line-of-sight velocity gradient across the cluster at the confidence level of $97 \%$. The identified dipole moment vector, showing the large scale velocity gradient, is pointing toward the east and does not coincide with that of the X-ray temperature gradient vector observed by $A S C A$, which is actually pointing toward the southeast.

ii) Spin Vector Distribution in the Virgo Cluster

We looked for any systematic tendency in the distribution of spin vectors of galaxies in the Virgo cluster. We examined the correlation in the 5D phase space for 60 galaxies in the Virgo cluster for which the distances derived from the Tully-Fisher relation and the spin vector orientations are known. We tentatively conclude that there is no striking correlation in the $5 \mathrm{D}$ phase space for this sample. It is important to check whether the apparent lack of correlation is consistent with each scenario of galaxy formation. Numerical studies to derive estimates on expected residual correlation from simulations of various scenarios are under way. 